\title{
Low back pain associates with altered activity of the cerebral cortex prior to arm movements that require postural adjustment
}

\author{
Jesse V. Jacobs ${ }^{1,2,3}$, Sharon M. Henry ${ }^{1,2}$, and Keith J. Nagle ${ }^{3}$ \\ ${ }^{1}$ Dept. of Rehabilitation and Movement Science, University of Vermont, Burlington, VT, USA \\ ${ }^{2}$ Dept. of Orthopaedics and Rehabilitation, University of Vermont, Burlington, VT, USA \\ ${ }^{3}$ Dept. of Neurology, University of Vermont, Burlington, VT, USA
}

\section{Abstract}

Objective: To determine whether low back pain (LBP) associates with altered postural stabilization and concomitant changes in cerebrocortical motor physiology.

\begin{abstract}
Methods: Ten participants with LBP and 10 participants without LBP performed self-initiated, voluntary arm raises. Electromyographic onset latencies of the bilateral internal oblique and erector spinae muscles were analyzed relative to that of the deltoid muscle as measures of anticipatory postural adjustments (APAs). Amplitudes of alpha event-related desynchronization (ERD) and of Bereitschaftspotentials (BP) were calculated from scalp electroencephalography as measures of cerebrocortical motor physiology.
\end{abstract}

Results: The APA was first evident in the trunk muscles contralateral to the arm raise for both groups. Significant alpha ERD was evident bilaterally at the central and parietal electrodes for participants with LBP but only at the electrodes contralateral and midline to the arm raise for those without LBP. The BP amplitudes negatively correlated with APA onset latencies for participants with (but not for those without) LBP.

Conclusions: Cerebrocortical activity becomes altered prior to arm movements requiring APAs for individuals with chronic LBP.

Significance: These results support a theoretical model that altered central motor neurophysiology associates with LBP, thereby implying that rehabilitation strategies should address these neuromotor impairments.

\section{Keywords}

back pain; anticipatory postural adjustment; posture; cerebral cortex; EEG

\footnotetext{
(c) 2009 International Federation of Clinical Neurophysiology. Published by Elsevier Ireland Ltd. All rights reserved.

Contact: Jesse V Jacobs, University of Vermont, 305 Rowell Building, 106 Carrigan Drive, Burlington, VT 05405; JJacobs@ uvm.edu, Phone: 802-656-8647, Fax: 802-656-6586.

Publisher's Disclaimer: This is a PDF file of an unedited manuscript that has been accepted for publication. As a service to our customers we are providing this early version of the manuscript. The manuscript will undergo copyediting, typesetting, and review of the resulting proof before it is published in its final citable form. Please note that during the production process errors may be discovered which could affect the content, and all legal disclaimers that apply to the journal pertain.

Conflicts of Interest: The authors have no conflicts of interest to disclose.
} 


\section{INTRODUCTION}

Seventy to 85 percent of people experience low back pain (LBP) and yearly expenses due to LBP are estimated to total 100 billion dollars in the United States (Andersson, 1999; Katz, 2006). Although estimates vary based on the population sampled, up to $85 \%$ of people with LBP experience chronic, recurrent symptoms (Andersson, 1999), demonstrating ineffective treatment for a large number of patients. Thus, we require a better understanding of the mechanisms that contribute to LBP in order to develop more effective rehabilitation strategies and improve treatment outcomes (Ebenbichler et al., 2001).

LBP has been traditionally referred to in terms of mechanical changes to the spine and its surrounding tissues (Adams, 2004). Alternatively, a 'kinesiopathologic' or 'functional pathology' model has recently been proposed, asserting that altered neural control of movement represents one possible mechanism for the development or persistence of chronic, recurrent LBP (Sahrmann, 2002; Adams, 2004; Langevin and Sherman, 2007). This model theorizes that repeated movements and prolonged postures during everyday activities contribute to low back symptoms and pathology because these repeated movement patterns continually expose lumbopelvic musculoskeletal tissue to low-magnitude loading in the same manner, thereby accelerating either deconditioning through under-utilization or cumulative tissue stress and microtrauma through over-utilization. This pain-inducing trauma is then suggested to affect central sensory processing, which in turn, continues a cycle of aberrant movement patterns.

With regard to the altered neural functions hypothesized by the kinesiopathologic model, altered cerebrocortical processing of sensory input has been demonstrated, such that people with LBP exhibit an altered amplitude and spatial topography of cerebrocortical somatosensory-evoked potentials to painful or non-painful stimuli (Flor et al., 1997; Wiech et al., 2000; Schneider et al., 2004; Siddall et al., 2006; Diers et al., 2007). Somatosensory-evoked potentials in response to pain stimuli are also modified by background muscle activation in participants with LBP, but not in participants without LBP, suggesting a change with LBP in the neural interactions of pain perception and motor output (Knost et al., 1999). Changes associated with LBP in cerebrocortical motor physiology during self-initiated movements, however, remain unidentified.

In support of the kinesiopathologic model's assertion of altered motor control with LBP, patients with LBP exhibit altered patterns of postural coordination during voluntary movements (Hodges and Richardson, 1999; Kuriyama and Ito, 2005; Shum et al., 2005a, 2005b; Kiesel et al., 2007; Mok et al., 2007), and behavioral modification of a patient's coordination patterns often decreases or eliminates that patient's symptoms of LBP compared to when performing the movements with self-selected coordination patterns (Van Dillen et al., 2007). In addition, people with LBP exhibit postural instability during challenging tasks of stance posture (Mok et al., 2004; Cacciatore et al., 2005; Leitner et al., 2007; Popa et al., 2007) and in response to externally induced perturbations to posture (Henry et al., 2006). The timing of muscle responses to postural perturbations has also been shown to predict future low back injury (Cholewicki et al., 2005). Despite this evidence to support the kinesiopathologic model, we are not aware of any studies that directly recorded changes in cerebrocortical motor physiology due to LBP during self-initiated voluntary movement. Thus, it remains necessary to determine whether changes in movement coordination associated with LBP also associate with changes in central motor neurophysiology in order to further validate the kinesiopathologic model.

Hodges and Richardson $(1996,1999)$ reported that patients with LBP exhibit delayed anticipatory postural adjustments (APAs) of the abdominal muscles during cued voluntary arm raises. APAs are defined as muscle activations within supporting body segments to stabilize and facilitate a voluntary limb movement against the anticipated perturbing forces resulting 
from the limb movement. The study of APAs provides unique insight into the central neural control of posture because APAs arise prior to when the perturbing forces resulting from the impending movement actually occur, thereby suggesting that APAs are pre-programmed by the central nervous system (Massion, 1992). Specific regions of the central nervous system involved in generating APAs include the supplementary and primary motor cortex (Viallet et al., 1992). Thus, delayed APA activity during movement in patients with LBP provides indirect support for the kinesiopathologic model's assertion that altered cerebrocortical motor physiology contributes to LBP.

Additional studies investigating the APAs of people with LBP demonstrated that their delayed APAs represent changes in coordination strategies rather than pain interference (Moseley and Hodges, 2005) or delayed neural transmission (Hodges, 2001). Further, people with LBP exhibit decreased anticipatory lumbopelvic movement in preparation for arm raises, which associates with increased spinal displacement during the arm raise (Mok et al., 2007). Therefore, in support of the kinesiopathologic model, chronic LBP associates with delays in the timing of APAs that are centrally generated and changes in preparatory lumbopelvic coordination that result in increased spinal displacements that would presumably accelerate cumulative tissue stress and microtrauma with repetition (Langevin and Sherman, 2007).

Pre-activation of the abdominal muscles during arm raises, however, is not always evident in people without a history of LBP, thereby causing uncertainty regarding the functional relevance of delayed APAs with LBP (Allison and Henry, 2002; Marshall and Murphy, 2003); could abdominal activation after movement onset in people without a history of LBP predispose those individuals to future LBP, or does it suggest a lack of necessity for pre-movement abdominal activation in order to maintain a life without LBP? Although inter-individual and inter-trial variability in APA onset latencies represent hallmarks of the behavior for people without a history of LBP, it should be noted that inter-trial variability decreases with both a history of chronic LBP and in response to experimentally induced LBP (Moseley and Hodges, 2006; Jacobs et al., 2009). In addition, the timing of the abdominal activation related to the APA correlates with the extent to which the area of that muscle's representation within the motor cortex becomes remodeled in people with LBP (Tsao et al., 2008). Thus, the change in APA onset latency appears to represent a functionally relevant change in central motor control related to the experience of LBP. This study, therefore, seeks to provide a correlation analysis in order to understand the mechanisms of the inter-individual variability that remain so enigmatic yet potentially important to our understanding of chronic LBP.

Although observations of delayed, less-variable APAs and related changes in motor neuroanatomy provide indirect evidence for an altered central neural control of movement with chronic LBP, no direct measures of central neurophysiology have been recorded to confirm the hypothesis that LBP associates with altered central motor control during self-initiated voluntary movement. The Bereitschaftspotential (BP) - a pre-movement electroencephalographic (EEG) potential characterized by a negative shift in the EEG signal before voluntary movements - represents a measure of cerebrocortical motor physiology related to movement preparation (Kornhuber and Deecke, 1964; Shibasaki and Hallett, 2006). In addition, the neural generators of the BP include the supplementary and primary motor cortex (Shibasaki and Hallett, 2006), thereby implicating the supplementary and primary motor cortex in the generation of both the BP and the APA. In support of this overlapping neurophysiology of the BP and the APA, the amplitude of the BP increases at electrodes overlying the supplementary and primary motor cortex when (1) the EEG signal is time-locked to the onset of the APA rather than to the prime movement (Saitou et al., 1996), and (2) the $\mathrm{BP}$ is compared between a movement that requires an APA to a movement that does not require an APA (Yoshida et al., 2008). In addition, transcranial magnetic stimulation of the primary motor cortex just prior to the onset of an APA (that is, when a BP would have presumably been 
evident) selectively facilitates the motor evoked potentials of a muscle used as part of the APA, but not of a muscle used as part of the goal-directed limb movement (MacKinnon et al., 2007). Thus, cerebrocortical motor preparation and, therefore, components of the BP likely relate specifically to the preparation of the APA. The BP would, therefore, provide insight into whether the delayed APAs that are evident with LBP associate with changes in central motor neurophysiology.

In addition to the BP, event-related desynchronization (ERD) of EEG signals within alpha (8to $13-\mathrm{Hz}$ ) frequencies provides another measure of movement-related cortical activation. Alpha ERD is defined by a decrease in alpha power just prior to movement onset (Neuper et al., 2006). Alpha ERD is coincident with increased cortico-cortical and cortico-spinal excitation of sensory-motor cortex (Rau et al., 2003), suggesting that alpha ERD represents cerebrocortical activation. When defined by the upper frequencies within the alpha band, alpha ERD exhibits a spatial topography that is specific to the somatotopic representation of cortex relevant to the movement task (Pfurtscheller et al., 2000). The BP and alpha ERD, however, exhibit different spatio-temporal characteristics, suggesting they are not two measures of the same underlying motor process (Babiloni et al., 1999; Shibasaki and Hallett, 2006). Although we are unaware of any studies examining alpha ERD in patients with chronic LBP, alpha ERD has been shown to increase when a movement is expected to correspond with an experimentally-induced painful stimulus, and alpha ERD amplitudes predict a participant's subsequent evaluation of the stimulus-induced pain intensity (Babiloni et al., 2005, 2006). Therefore, in addition to investigating changes in the BP in people with chronic LBP, analyzing the alpha ERD may also provide insight into potential LBP-related changes in cerebrocortical motor neurophysiology.

The kinesiopathologic model of LBP remains untested regarding whether altered movement patterns represent a pre-existing condition for the development of LBP or if such changes in motor control simply contribute to the persistence or recurrence of LBP after an initial injury. The latter contribution, however, remains important for discerning why some individuals develop chronic LBP while others do not, and is supported by observations that (1) normalizing the altered movement patterns performed by people with chronic LBP decreases or resolves the movement-induced LBP (Van Dillen et al., 2007), and (2) altered motor patterns remain evident from people with a history of LBP who do not exhibit pain at the time of testing (Hodges and Richardson, 1999; Jacobs et al., 2009) as well as when people without a history of LBP perform several trials after the cessation of acute, experimentally induced LBP (Moseley and Hodges, 2005, 2006). Thus, the kinesiopathologic model would support the hypothesis that people with chronic LBP exhibit altered cerebrocortical motor neurophysiology consequent to long-term changes in postural coordination that persist independent of pain state but which contribute to the persistence or recurrence of pain.

To test the hypothesis that chronic LBP associates with altered cerebrocortical motor neurophysiology, we recorded the BPs, alpha ERD, and APAs of participants with and without LBP during voluntary, self-initiated arm raises. We predicted that participants with LBP would exhibit delayed APAs (Hodges and Richardson, 1996, 1999), altered BP amplitudes, and significant alpha ERD at electrodes overlying not only sensory-motor cortex of muscles activated for the arm movement, but also at those overlying the muscles activated for the APA. We further predicted these changes would be independent of the participants' pain state, as these changes in motor control likely represent long-term adaptive changes in motor strategy, as opposed to a temporary modification in response to current pain. Identifying changes in cerebrocortical motor physiology in people with LBP would validate a potential paradigm shift in the assessment and management of LBP to focus on aspects of motor control impairments in addition to the impairments of the lumbopelvic musculoskeletal structures. Such a paradigm 
shift would stimulate further study to investigate the effects of targeted movement re-education in order to improve treatment outcomes.

\section{METHODS}

\section{Participants}

Ten participants with chronic-recurrent, non-specific, mechanical LBP and 10 participants without LBP gave written informed consent in compliance with the Declaration of Helsinki to participate in the protocol, which was approved by the Institutional Review Board of the University of Vermont. Participants with and without LBP were differentiated by a history of pain in the lumbar area that has impaired at least three activities of daily living for a minimum of 12 months. Based on an intake questionnaire and/or clearance by the referring physician or physical therapist, participants with LBP were included if they reported being active in work, homemaking, or school, and were not in litigation or seeking/receiving compensation for their LBP. Further, other than LBP, all participants reported no history of neurological,

cardiovascular, respiratory, or psychiatric disorders, and they reported no history or diagnosis of neuromuscular or joint disorders, dizziness, diabetes, systemic infection, alcoholism, tumor or suspected carcinoma, surgery in the previous three months, any back surgery, spinal fracture or dislocation, herniated disc, stenosis, kyphosis, scoliosis, injections to relieve back pain in the previous 7 days, as well as pain, numbness or tingling below the knee.

These exclusion criteria were implemented in order to (1) focus on a sub-population of chronic, recurrent LBP for which there is limited understanding, and (2) strengthen confidence that any group differences in this cross-sectional study were due to the LBP and not another factor known to confound posture control or cerebrocortical neurophysiology. We note, however, that these restrictions on our subject sample limit the generalizability of this study's results to only this sub-population of chronic, recurrent LBP.

\section{Protocol}

The task was to voluntarily raise the dominant arm as fast as possible while seated (two participants with LBP and one participant without LBP raised their left arm). Prior to performing the task, the participants responded to two questionnaires that quantified their pain and disability: the 0-10 Numeric Pain Rating Scale (Childs et al., 2005), and the Modified Oswestry Disability Questionnaire (Fritz and Irrgang, 2001). Electrodes were then attached for EEG, electromyographic (EMG), and electrooculographic (EOG) recordings. To record the $\mathrm{BP}$ and alpha ERD, silver/silver-chloride electrodes were affixed to the scalp at standard locations described by the 10/20 system (Jasper, 1958). In addition, an electrode at FCz of the 10/10 system (Chatrian et al., 1985) was added to enhance recording coverage over the frontocentral region, where the BP is most evident (Shibasaki and Hallett, 2006). The EOG electrodes were placed above the left outer canthus and below the right outer canthus to identify and remove any trials with eye-movement artifacts that confound EEG signals. The EEG signals were referenced to linked electrodes placed on the earlobes.

To record the timing of muscle activations related to the arm raise, double-differential surface EMG electrodes were placed $2 \mathrm{~cm}$ apart along the length of the contracted muscle belly of the dominant arm's anterior deltoid muscle (DELT; a shoulder muscle activated for raising the arm) as well as bilaterally over the internal oblique muscles (IO; abdominal muscles used as part of the APA to stabilize the trunk when the arm is raised) and erector spinae muscles (ESP; back muscles that are also used as part of the APA to stabilize the trunk when the arm is raised). Electrodes over the IO muscles were placed $2.5 \mathrm{~cm}$ medial and $2.5 \mathrm{~cm}$ cephalad to the anteriorsuperior iliac spine, and those for the ESP muscles were placed $2.5 \mathrm{~cm}$ lateral to the third lumbar segment of the spine. Although we recognize that surface EMG activity over the IO likely also 
reflects activity of the underlying transversus abdominis, both muscles become similarly delayed for participants with chronic LBP during a rapid arm raise task (Hodges and Richardson, 1999). Therefore, for simplicity, we will refer to this EMG electrode position only in terms of the more superficial IO muscle. Because APA characteristics depend on the speed of the arm raise (Hodges and Richardson, 1999), a tri-axial accelerometer (NeuwGhent Technology, Lagrangeville, NY) was affixed at the dorsal midpoint between the distal end of the ulna and radius of the participants' dominant arm to record the acceleration of the arm's movement. An accelerometer was also affixed just below the sternal notch of the manubrium to record the accelerations of the participants' trunk movements as measures of how well the APA stabilized trunk orientation.

When performing arm raises, the participants sat upright on a stable, adjustable stool with their back unsupported, feet on the floor, arms at their sides, and with their gaze fixed on a visual target placed two meters in front of them at eye level. Joint angles were measured to establish 90-degree flexion at the hip and knee as well as zero-degree plantar-/dorsiflexion at the ankle in order to ensure similar postural orientations across the participants. This initial position was intermittently verified during testing by visual inspection with a goniometer. The participants were instructed to fixate their gaze on the target without blinking their eyes for at least three seconds prior to raising their arm in order to minimize eye-movement artifacts in the EEG signal. If the EEG signals appeared to exhibit EMG artifact during data collection, the participant was instructed to relax their jaw or forehead. The participants were also instructed to exhale and relax their abdomen prior to raising their arm in order to (1) prevent APA activity associated with breathing from confounding the APA activity associated with the arm raise, and (2) minimize background EMG activation, which also facilitated the identification of muscle onset times. Background EMG activation was monitored online and, if necessary, the participants were reminded to relax their abdominal or back muscles in order to maintain low levels of background EMG activity.

With the participants' arms initially positioned in approximately zero degrees of both glenohumeral flexion and abduction, they flexed their dominant arm 90 degrees as fast as they could to a horizontal, outstretched position in front of them, and then returned the arm to its initial position. The participants raised their arm at a self-initiated pace (without cues or prompting) of approximately once every 10 seconds for a total of 125 trials. Hardware limitations prevented the simultaneous recording of arm accelerations and EMG activations. Therefore, the participants performed one set of 25 trials while recording the accelerometer signals, a second set of 75 trials while recording EMG signals, and then a third set of 25 trials while recording the accelerometer signals. The participants were not made aware of the differences in the purpose of each trial set. The participants rested, at minimum, every 25 trials and were instructed to ask for rest when needed to prevent fatigue or soreness.

\section{Data Collection and Processing}

The level of the participants' LBP on the day of testing was quantified just prior to performing the protocol by the participants' responses to the Numeric Pain Rating Scale: an integer scale that asks the participants to rate the level of their back pain from zero to 10, zero representing no pain and 10 representing maximal pain. The level of the participants' disability was also determined by their responses to the Modified Oswestry Disability Questionnaire. This questionnaire consists of 10 items related to aspects of daily living and asks the participants to identify the extent to which their back pain affects these aspects of daily living on a 5-item ordinal scale. The participants' disability was thus quantified as a percent of maximum disability by summating the responses from each item's ordinal scale, dividing the sum score by a maximum score of 50 and then multiplying the result by 100 percent. 
Continuous EEG, EOG, and EMG signals were recorded during the second trial set of 75 arm raises from a DUO amplifier using Harmonie software (Stellate, Montreal, Canada). Data were sampled at $1000 \mathrm{~Hz}$ with 16-bit resolution and amplified by 1000. The EEG, EOG, and EMG signals were band-pass filtered at $0.07-60 \mathrm{~Hz}, 0.1-60 \mathrm{~Hz}$, and 30-400 Hz, respectively. Input impedance was held under $5 \mathrm{k}$ for the EEG and EOG signals and held under $10 \mathrm{k}$ for the EMG signals. Signals from the accelerometers were recorded from a direct-current interface box into the DUO amplifier during the first and third trial sets of $25 \mathrm{arm}$ raises. These accelerometer signals were recorded at $200 \mathrm{~Hz}$ with 16-bit resolution, amplified by 1000 and band-pass filtered at $0.07-25 \mathrm{~Hz}$.

The data were subsequently reduced and analyzed offline using Matlab software (MathWorks, Inc., Natick, MA). The continuous data were first spliced into 6-s trials, with three seconds before and three seconds after the activation onset of the IO muscle contralateral to the arm being raised. Trials were time-locked to this onset because the contralateral IO muscle consistently represented the first activated muscle of the APA, and previous reports suggest that components of BP activity that are related to the APA can be captured when time-averaging the data to the onset of the APA (Saitou et al., 1996). The EEG data were further reduced so as to focus the analysis on the electrode locations known to exhibit maximal BP amplitudes and alpha ERD; the analysis on BP amplitudes was constrained to the F3, Fz, F4, FCz, C3, Cz, $\mathrm{C} 4, \mathrm{P} 3, \mathrm{Pz}$, and $\mathrm{P} 4$ electrodes, and the analysis on alpha ERD was constrained to the $\mathrm{C} 3, \mathrm{Cz}$, C4, P3, Pz, and P4 electrodes (Shibasaki and Hallett, 2006; Neuper et al., 2006). For those participants who performed the task with their left arm, the lateral identification of EEG electrodes was reversed such that group data could be referred in terms of electrodes placed ipsilateral or contralateral to the arm movement. Thus, notation for the F3, C3, P3, F4, C4 and $\mathrm{P} 4$ electrodes changes to $\mathrm{Fc}, \mathrm{Cc}$, and $\mathrm{Pc}$ for electrodes positioned contralateral to the arm movement, as well as to $\mathrm{Fi}, \mathrm{Ci}$, and $\mathrm{Pi}$ for electrodes positioned ipsilateral to the arm movement.

Trials with eye-movement or EMG artifact in the EEG were removed if the EOG or EEG signals exceeded $\pm 50 \mu \mathrm{V}$ of displacement during the three seconds that preceded the activation onset of the contralateral IO muscle. The analysis of all variables was subsequently performed on the first 40 artifact-free trials recorded during the second trial set of 75 trials. Baseline activity (defined as the average EEG voltage from 3000 to $2900 \mathrm{~ms}$ before activation onset of the IO muscle contralateral to the arm movement) was subtracted from each trial's EEG voltage signal. Average EEG traces were then computed from the first 40 artifact-free trials recorded during the second trial set of 75 trials. Mean BP amplitudes were defined from each participant's average EEG waveform as the mean voltage amplitude over the final $100 \mathrm{~ms}$ that preceded the onset of the contralateral IO muscle (Fig. 1).

For calculating alpha ERD (Pfurtscheller and Lopes da Silva, 1999), the EEG signals were digitally re-referenced to a common average reference that included every cephalic electrode. Continuous morlet wavelet transforms (within the alpha frequencies of $8-13 \mathrm{~Hz}$ ) were performed on each of the 40 artifact-free trials. These morlet coefficients of alpha EEG were rectified and averaged by participant. To account for individual differences in alpha oscillations, each participant's peak individual alpha frequency (IAF) was determined by identifying the wavelet scale (corresponding to a pseudo-frequency within the alpha band) at which maximum morlet coefficients were evident. Power within the upper alpha band was then determined from scales representing the IAF to the IAF $+2 \mathrm{~Hz}$. The morlet coefficients were then averaged within these upper alpha frequencies, and the time-varying alpha coefficients were normalized to a percentage change in alpha power from a baseline average calculated from 2500 to $2000 \mathrm{~ms}$ before activation onset of the contralateral IO muscle. Mean coefficients were then binned over the four subsequent 500-ms intervals that preceded the activation onset of the contralateral IO muscle (Fig. 1). The size and speed of the arm raises elicited eye blinks 
following movement onset, thereby precluding the analysis of movement-related EEG activity after movement onset.

Muscle onset times were visually selected from an interactive graphing program that displayed the rectified EMG traces relative to a threshold activation of 3 standard deviations above the mean baseline activity of the 6-s epoch's first $500 \mathrm{~ms}$. The participant's group assignment was not identified when selecting onset times. Muscle onset times were selected for each trial, and those of the DELT muscle were subtracted from those of the IO and ESP muscles to generate the APA onset latencies of the IO and ESP muscles. These APA onset latencies were then averaged by participant for analysis. For clarity, Figure 1 illustrates the relative timing of the selected EEG and EMG measures.

Given APA activation depends on the velocity of the associated movement (Hodges and Richardson, 1999; Bouisset et al., 2000; Bleuse et al., 2005), maximum velocities of the arm were determined for each trial from the integral of the arm's tangential acceleration in the sagittal plane. In addition, maximum velocities of trunk movement induced by the arm raises were determined in the sagittal and frontal planes. The maximum arm and trunk velocities of these individual trials were then averaged by participant for analysis.

\section{Statistical Analysis}

Due to violations of the assumptions that underlie parametric statistics, all analyses were performed using non-parametric statistics. Bonferroni corrections were applied to alleviate the potential for type I errors due to multiple comparisons. Differences between the groups with and without LBP in mean IO and ESP muscle onset latencies, mean BP amplitudes, baseline morlet coefficients of upper alpha power, questionnaire scores, demographic characteristics, as well as arm and trunk velocities were analyzed by Mann-Whitney $\mathrm{U}$ tests. To determine the existence of significant alpha ERD at each electrode and for each group, Friedman's ANOVA compared the mean normalized alpha coefficients over the four 500-ms intervals that preceded the activation of the contralateral IO muscle. To determine whether the participants' cerebrocortical motor physiology was associated with their APA coordination, Spearman's correlation coefficients were performed for each group, comparing the APA latencies of the IO and ESP muscles with the participants' mean BP amplitudes from the electrode of maximal $\mathrm{BP}$ amplitude $(\mathrm{Cz})$. To determine if the cerebrocortical motor physiology of the participants with LBP associates with their reported pain and disability, Spearman's correlation coefficients were calculated among the participants' questionnaire scores and their BP as well as alpha ERD amplitudes.

\section{RESULTS}

The groups were matched for gender and were of similar age [Mann-Whitney $\mathrm{Z}=0.91, \mathrm{P}=$ $0.36]$, height $[Z=0.61, P=0.54]$, and weight $[Z=1.14, P=0.26]$ (Table 1). Participants with LBP were not in an acute symptom flare-up and reported mild levels of pain and disability at the time of testing (Table 1). Even so, the group with LBP expectedly reported higher levels of pain on the Numeric Pain Rating Scale $[Z=2.71, P=0.0066]$ and higher levels of disability on the Modified Oswestry Disability Questionnaire than the group without LBP $[\mathrm{Z}=3.69, \mathrm{P}$ $=0.00023$ ].

Although, on average, the group with LBP exhibited delayed APA onset latencies compared to the group without LBP (Fig. 2), these differences were not statistically significant either before or after Bonferroni correction [contralateral IO: $\mathrm{Z}=1.32$, Bonferroni-corrected $\mathrm{P}=0.76$; ipsilateral IO: $Z=0.04$, Bonferroni-corrected $P=1.0$; contralateral $E S P: Z=1.89$, Bonferronicorrected $\mathrm{P}=0.24$; ipsilateral ESP: $\mathrm{Z}=1.32$, Bonferroni-corrected $\mathrm{P}=0.76]$. Likewise, on average, the group with LBP exhibited larger (more negative) BP amplitudes than the group 
without LBP, but these differences also were not significant over any electrode, either before or after Bonferroni correction [range of $Z=0.19-1.44$, Bonferroni-corrected $P=1.0$ ] (Fig. 3). For both groups, mean BP amplitudes were largest at the $\mathrm{Cz}$ electrode (Fig. 3).

Although BP amplitudes were not significantly different between those with and without LBP, onset latencies of the contralateral ESP muscle significantly correlated with BP amplitudes for the group with LBP but not for the group without LBP (Fig. 4). Specifically, the relationship between BP amplitudes and APA onset latencies was such that earlier APA onset latencies were associated with larger BP amplitudes, and more delayed APA onset latencies were associated with smaller BP amplitudes for the group with LBP. The BP amplitudes of the group with LBP were not significantly correlated at any electrode with questionnaire scores of pain or disability, either before or after Bonferroni correction [range of Spearman's $r=0.017-0.57$, Bonferroni-corrected $\mathrm{P}=1.0]$.

As illustrated in Fig. 5, significant alpha ERD was evident prior to Bonferroni correction for the group with LBP at all six central and parietal electrodes; after Bonferroni correction, the significance of the alpha ERD for the group with LBP remained clearly evident at $\mathrm{Cc}, \mathrm{Pc}$, and $\mathrm{Ci}$, but not at $\mathrm{Cz}, \mathrm{Pz}$, or Pi [Cc: Friedman's $\chi^{2}=21.24$, Bonferroni-corrected $\mathrm{P}=0.0005$; $\mathrm{Cz}$ : $\chi^{2}=8.03$, Bonferroni-corrected $\mathrm{P}=0.27 ; \mathrm{Ci}: \chi^{2}=12.94$, Bonferroni-corrected $\mathrm{P}=0.03$; Pc: Friedman's $\chi^{2}=13.08$, Bonferroni-corrected $\mathrm{P}=0.02$; Pz: $\chi^{2}=9.00$, Bonferroni-corrected $\mathrm{P}$ $=0.17$; Pi: $\chi^{2}=8.42$, Bonferroni-corrected $\left.\mathrm{P}=0.23\right]$. For the group without LBP, significant alpha ERD was evident prior to Bonferroni correction at $\mathrm{Cc}, \mathrm{Cz}, \mathrm{Pc}$, and $\mathrm{Pz}$ but not at $\mathrm{Ci}$ or $\mathrm{Pi}$; after Bonferroni correction, significant alpha ERD was evident only at $\mathrm{Cz}$ and $\mathrm{Pc}$ [Cc: Friedman's $\chi^{2}=9.22$, Bonferroni-corrected $\mathrm{P}=0.16$; Cz: $\chi^{2}=13.36$, Bonferroni-corrected $\mathrm{P}$ $=0.02 ; \mathrm{Ci}: \chi^{2}=4.24$, Bonferroni-corrected P $=1.0$; Pc: Friedman's $\chi^{2}=12.89$, Bonferronicorrected P $=0.03$; Pz: $\chi^{2}=10.93$, Bonferroni-corrected P $=0.07$; Pi: $\chi^{2}=4.77$, Bonferronicorrected $\mathrm{P}=1.0]$. It is worthy to note that statistically insignificant results were evident at electrodes exhibiting larger average alpha ERD than some electrodes found to exhibit significant alpha ERD, but the inter-individual variability was greater at the electrodes that were statistically insignificant (Fig. 5). The alpha ERD amplitudes of the group with LBP were not significantly correlated at any electrode with questionnaire scores of pain or disability, either before or after Bonferroni correction [range of Spearman's $r=0.00-0.56$, range of Bonferroni-corrected $\mathrm{P}=0.72-1.0]$. Peak IAFs and baseline morlet coefficients at all six electrodes were similar between the groups with and without LBP [range of Mann-Whitney Z $=0.037-0.87$, uncorrected $\mathrm{P}=0.38-0.97]$.

Table 2 provides the average maximum velocities of the arm and trunk for the groups with and without LBP. The maximum velocities of the participants' arm raises were statistically similar between those with and without LBP, either before or after Bonferroni correction [trial set one: $\mathrm{Z}=0.64$, Bonferroni-corrected $\mathrm{P}=1.0$; trial set three: $\mathrm{Z}=0.87$, Bonferroni-corrected $\mathrm{P}=0.77]$. The maximum velocities of the trunk were statistically similar among those with and without LBP, either before or after Bonferroni correction [sagittal velocity: $\mathrm{Z}=0.12$, Bonferronicorrected $\mathrm{P}=1.0$ for trial set one and $\mathrm{Z}=0.08$, Bonferroni-corrected $\mathrm{P}=1.0$ for trial set three; frontal velocity: $\mathrm{Z}=0.70$, Bonferroni-corrected $\mathrm{P}=0.98$ for trial set one and $\mathrm{Z}=0.82$, Bonferroni-corrected $\mathrm{P}=0.82$ for trial set three].

\section{DISCUSSION}

The results demonstrate that the participants with LBP exhibited a significant correlation between their BP amplitudes and the APA onset latencies of their contralateral ESP muscle, which was not evident from the participants without LBP. In addition, the group with LBP exhibited a different spatial topography of significant alpha ERD than those without LBP. Arm and trunk velocities demonstrated no evidence that the participants with LBP exhibited a 
protective (slowed) movement strategy, greater fatigue, or increased trunk movement compared to the participants without LBP. Taken together, these observations are the first to suggest that individuals with chronic LBP exhibit altered cerebrocortical activity prior to selfinitiated, voluntary arm movements that include APAs.

It remains unclear whether the difference in the alpha ERD's spatial topography between the participants with and without LBP represent (1) activation of the same somatotopic functional regions that are of different area, or (2) activation of different somatotopic functional regions. The difference in the spatial topography of the groups' alpha ERD is consistent, however, with previous reports of altered sensory-motor topography within the lumbar and abdominal representations of participants with LBP (Flor et al., 1997; Tsao et al., 2008). Specifically, using transcranial magnetic stimulation, the area of the primary motor cortex that elicits activation of the abdominal musculature increases and is centered more laterally for individuals with chronic LBP than for those without LBP (Tsao et al., 2008). Our task elicited activation of the IO and ESP muscles bilaterally (with activation contralateral to arm movement preceding that ipsilateral to the arm movement). Thus, alpha ERD related to the cortical activation of these muscles' somatotopic representations might be expected to spread and lateralize, as demonstrated by bilaterally significant alpha ERD for participants with LBP but at midline and contralateral electrodes for those without LBP. The results, therefore, most closely support an interpretation that the group differences in the spatial topography of alpha ERD reflect activation of the same somatotopic functional regions that are of different areas.

The significant correlation between the APA onset latencies of the contralateral ESP muscle and BP amplitudes of the group with, but not without, LBP may additionally reflect this change in somatotopy. Although speculative, the functional significance of this correlation may reflect a more direct influence of executive motor centers on the coordination of posture and movement for individuals with LBP, whereas this coordination has been suggested to be automated for individuals without history of LBP (Tsao et al., 2009), such that sub-cortical centers known to contribute to the integration of the APA and prime movement (Schepens and Drew, 2004) perhaps exert greater influence on the coordination of the APA for people without LBP. Compensation by increased cerebrocortical influence on postural coordination may also explain why we did not observe a statistically significant difference in APA latencies between the groups with and without LBP. It is possible that the self-initiated arm raises of our study permitted the time necessary for the activation of the cerebral cortex to compensate for delayed APAs, whereas such a compensation was not permitted in the reports by Hodges and Richardson $(1996,1999)$ because they studied arm raises in response to unpredictably timed cues.

Although significant correlations among the participants' pain and disability due to LBP with their BP amplitudes or alpha ERD would confirm that group differences reflect changes due to LBP, our results are consistent with the interpretations of previous studies that the changes in motor control evident from people with chronic LBP represent a long-term adoption of altered motor strategies rather than an acute change related to momentary pain (Hodges and Richardson, 1999; Hodges, 2001). Specifically, this study's observed changes in cortical motor neurophysiology occurred in a sample of participants with chronic LBP who were not experiencing an acute flare-up of LBP and reported low levels of pain at the time of testing. In addition, alpha ERD as well as BP amplitudes were not correlated to the participants' reported levels of pain or disability just prior to testing. Therefore, our results concur with previous studies that demonstrated (1) delayed and less variable APA onset latencies in a sample of patients with a history of recurrent LBP who were in a quiescent period of pain (Hodges and Richardson, 1999; Jacobs et al., 2009), and (2) a sustained decrease in the variability of APA onset times many trials after the cessation of acute, experimentally induced LBP (Moseley and Hodges, 2006). The dissociation of the participants' altered APAs and cerebrocortical motor 
neurophysiology from their pain at the time of testing thus supports the interpretation of previous studies that these changes in the motor control of people with chronic LBP represent long-term changes in motor strategies rather than modifications in response to momentary pain.

Identifying changes in motor neurophysiology in patients with LBP has significant clinical ramifications. To date, the rehabilitation of people with LBP is often unsuccessful, leading to chronic, unresolved pain or recurrent episodes of pain (Croft, 1997; Andersson, 1999). The need for more successful rehabilitation strategies may be rooted in the need for a better understanding of the mechanisms that contribute to an individual's initial and recurrent LBP. Whereas chronic LBP has long been treated from a mechanical perspective, recent studies have built support for a kinesiopathologic model, asserting that altered movement coordination contributes to tissue pathology and LBP (Sahrmann, 2002; Adams, 2004; Langevin and Sherman, 2007). As a cross-sectional study, this study cannot elucidate the contribution of altered movement patterns to initial low back injury. This study's results, however, demonstrate changes in the central motor neurophysiology of participants with recurrent LBP while in a quiescent period of pain, thereby suggesting that altered central motor control may at least contribute to the persistence or recurrence of LBP. This study, therefore, provides support for the rehabilitation of chronic, recurrent LBP to include re-training of postural coordination (Tsao and Hodges, 2008; Sahrmann 2002).

\section{Acknowledgments}

We thank our research volunteers for their participation. We also thank Sarah Goodrich, BS, R EEG T for help with EEG preparation and data collection, Katherine M. Schneider, BS for help with data processing, Andrea L. Trombley, MPT and Theresa M. Miner, BS, R EEG T for recruitment and scheduling assistance, Juvena R. Hitt, BS for hardware assistance, and Lori J. Fontneau, RN for administrative assistance. We thank Fletcher Allen Health Care, Timberlane Physical Therapy, Dee Physical Therapy, and Evolution Physical Therapy of the Burlington, VT area for recruitment assistance.

This research was supported by National Institutes of Health / National Institute of Arthritis and Musculoskeletal and Skin Diseases [T32AR07568-09 to J. J.], a University of Vermont College of Nursing and Health Sciences Research Incentives Grant to S. H., and a University of Vermont Undergraduate Research Endeavors Competitive Award to S. H.

\section{REFERENCES}

Adams MA. Biomechanics of back pain. Acupunct Med 2004;22:178-188. [PubMed: 15628775]

Alegre M, Labarga A, Gurtubay IG, Iriarte J, Malanda A, Artieda J. Movement-related changes in cortical oscillatory activity in ballistic, sustained and negative movements. Exp Brain Res 2003;148:17-25. [PubMed: 12478393]

Allison GT, Henry SM. The influence of fatigue on trunk muscle responses to sudden arm movements, a pilot study. Clin Biomech 2002;17:414-417.

Andersson GB. Epidemiological features of chronic low-back pain. Lancet 1999;354:581-585. [PubMed: 10470716]

Babiloni C, Brancucci A, Capotosto P, Arendt-Nielsen L, Chen AC, Rossini PM. Expectancy of pain is influenced by motor preparation: a high-resolution EEG study of cortical alpha rhythms. Behav Neurosci 2005;119:503-511. [PubMed: 15839796]

Babiloni C, Brancucci A, Del Percio C, Capotosto P, Arendt-Nielsen L, Chen AC, Rossini PM. Anticipatory electroencephalography alpha rhythm predicts subjective perception of pain intensity. $\mathrm{J}$ Pain 2006;7:709-717. [PubMed: 17018331]

Babiloni C, Carducci F, Cincotti F, Rossini PM, Neuper C, Pfurtscheller G, Babiloni F. Human movement-related potentials vs desynchronization of EEG alpha rhythm: a high- resolution EEG study. Neuroimage 1999;10:658-665. [PubMed: 10600411]

Bleuse S, Cassim F, Blatt JL, Defebvre L, Derambure P, Guieu JD. Vertical torque allows recording of anticipatory postural adjustments associated with slow, arm raising movements. Clin Neurophysiol 2005;20:693-699. 
Bouisset S, Richardson J, Zattara M. Do anticipatory postural adjustments occurring in different segments of the postural chain follow the same organisational rule for different task movement velocities, independently of the inertial load value? Exp Brain Res 2000;132:79-86. [PubMed: 10836638]

Cacciatore TW, Horak FB, Henry SM. Improvement in automatic postural coordination following alexander technique lessons in a person with low back pain. Phys Ther 2005;85:565-578. [PubMed: 15921477]

Chatrian GE, Lettich E, Nelson PL. Ten percent electrode system for topographic studies of spontaneous and evoked EEG activity. Am J EEG Technol 1985;25:83-92.

Childs JD, Piva SR, Fritz JM. Responsiveness of the numeric pain rating scale in patients with low back pain. Spine 2005;30:1331-1334. [PubMed: 15928561]

Cholewicki J, Silfies SP, Shah RA, Greene HS, Reeves NP, Alvi K, Goldberg B. Delayed trunk muscle reflex responses increase the risk of low back injuries. Spine 2005;30:2614-2620. [PubMed: 16319747]

Croft, P. Low back pain. In: Stevens, A.; Raftery, J., editors. Health Care Needs Assessment. Radcliffe Medical Press Oxford; New York: 1997. p. 129

Diers M, Koeppe C, Diesch E, Stolle AM, Hölzl R, Schiltenwolf M, van Ackern K, Flor H. Central processing of acute muscle pain in chronic low back pain patients: an EEG mapping study. J Clin Neurophysiol 2007;24:76-83. [PubMed: 17277582]

Ebenbichler GR, Oddsson LI, Kollmitzer J, Erim Z. Sensory-motor control of the lower back: implications for rehabilitation. Med Sci Sports Exerc 2001;33:1889-1898. [PubMed: 11689740]

Flor H, Braun C, Elbert T, Birbaumer N. Extensive reorganization of primary somatosensory cortex in chronic back pain patients. Neurosci Lett 1997;224:5-8. [PubMed: 9132689]

Fritz JM, Irrgang JJ. A comparison of a modified Oswestry Low Back Pain Disability Questionnaire and the Quebec Back Pain Disability Scale. Phys Ther 2001;81:776-788. [PubMed: 11175676]

Henry SM, Hitt JR, Jones SL, Bunn JY. Decreased limits of stability in response to postural perturbations in subjects with low back pain. Clin Biomech 2006;21:881-892.

Hodges PW. Changes in motor planning of feedforward postural responses of the trunk muscles in low back pain. Exp Brain Res 2001;141:261-266. [PubMed: 11713638]

Hodges PW, Richardson CA. Inefficient muscular stabilization of the lumbar spine associated with low back pain. A motor control evaluation of transversus abdominis. Spine 1996;21:2640-2650. [PubMed: 8961451]

Hodges PW, Richardson CA. Altered trunk muscle recruitment in people with low back pain with upper limb movement at different speeds. Arch Phys Med Rehabil 1999;80:1005-1012. [PubMed: 10489000]

Jacobs JV, Henry SM, Nagle KJ. People with chronic low back pain exhibit decreased variability in the timing of their anticipatory postural adjustments. Behav Neurosci 2009;123:455-458. [PubMed: 19331469]

Jasper HH. The ten twenty electrode system of the international federation. Clin Neurophysiol 1958;10:371-375.

Katz JN. Lumbar disc disorders and low-back pain: socioeconomic factors and consequences. J Bone Joint Surg Am 2006;88:21-24. [PubMed: 16595438]

Kiesel KB, Underwood FB, Mattacola CG, Nitz AJ, Malone TR. A comparison of select trunk muscle thickness change between subjects with low back pain classified in the treatment based classification system and asymptomatic controls. J Orthop Sports Phys Ther 2007;37:596-607. [PubMed: 17970406]

Knost B, Flor H, Birbaumer N, Schugens MM. Learned maintenance of pain: muscle tension reduces central nervous system processing of painful stimulation in chronic and subchronic pain patients. Psychophysiology 1999;36:755-764. [PubMed: 10554589]

Kornhuber HH, Deecke L. Hirnpotentialänderungen beim Menschen vor und nach Willkürbewegungen, dargestellt mit Magnetbandspeicherung und Rückwärtsanalyse. Pflügers Arch Eur J Physiologie 1964;281:52.

Kuriyama N, Ito H. Electromyographic functional analysis of the lumbar spinal muscles with low back pain. J Nippon Med Sch 2005;72:165-173. [PubMed: 16046833] 
Lamoth CJ, Stins JF, Pont M, Kerckhoff F, Beek PJ. Effects of attention on the control of locomotion in individuals with chronic low back pain. J Neuroeng Rehabil 2008;5:13. [PubMed: 18439264]

Langevin HM, Sherman KJ. Pathophysiological model for chronic low back pain integrating connective tissue and nervous system mechanisms. Med Hypotheses 2007;68:74-80. [PubMed: 16919887]

Leitner C, Mair P, Paul B, Wick F, Mittermaier C, Sycha T, Ebenbichler G. Reliability of posturographic measurements in the assessment of impaired sensorimotor function in chronic low back pain. $\mathrm{J}$ Electromyogr Kinesiol. 2007 Electronic Publication: November 17.

MacKinnon CD, Bissig D, Chiusano J, Miller E, Rudnick L, Jager C, Zhang Y, Mille ML, Rogers MW. Preparation of anticipatory postural adjustments prior to stepping. J Neurophysiol 2007;97:43684379. [PubMed: 17460098]

Marshall P, Murphy B. The validity and reliability of surface EMG to assess the neuromuscular response of the abdominal muscles to rapid limb movement. J Electromyogr Kinesiol 2003;13:477-489. [PubMed: 12932422]

Massion J. Movement, posture and equilibrium: interaction and coordination. Prog Neurobiol 1992;38:35-56. [PubMed: 1736324]

Mok NW, Brauer SG, Hodges PW. Hip strategy for balance control in quiet standing is reduced in people with low back pain. Spine 2004;29:E107-E112. [PubMed: 15014284]

Mok NW, Brauer SG, Hodges PW. Failure to use movement in postural strategies leads to increased spinal displacement in low back pain. Spine 2007;32:E537-E543. [PubMed: 17762795]

Moseley GL, Hodges PW. Are the changes in postural control associated with low back pain caused by pain interference? Clin J Pain 2005;21:323-329. [PubMed: 15951650]

Moseley GL, Hodges PW. Reduced variability of postural strategy prevents normalization of motor changes induced by back pain: a risk factor for chronic trouble? Behav Neurosci 2006;120:474-476. [PubMed: 16719709]

Neuper C, Wörtz M, Pfurtscheller G. ERD/ERS patterns reflecting sensorimotor activation and deactivation. Prog Brain Res 2006;159:211-222. [PubMed: 17071233]

Pfurtscheller G, Lopes da Silva FH. Event-related EEG/MEG synchronization and desynchronization: basic principles. Clin Neurophysiol 1999;110:1842-1857. [PubMed: 10576479]

Pfurtscheller G, Neuper C, Krausz G. Functional dissociation of lower and upper frequency mu rhythms in relation to voluntary limb movement. Clin Neurophysiol 2000;111:1873-1879. [PubMed: 11018505]

Popa T, Bonifazi M, Della Volpe R, Rossi A, Mazzocchio R. Adaptive changes in postural strategy selection in chronic low back pain. Exp Brain Res 2007;177:411-418. [PubMed: 16977448]

Rau C, Plewnia C, Hummel F, Gerloff C. Event-related desynchronization and excitability of the ipsilateral motor cortex during simple self-paced finger movements. Clin Neurophysiol 2003;114:1819-1826. [PubMed: 14499743]

Sahrmann, SA. Diagnosis and treatment of movement impairment syndromes. Mosby; St. Louis: 2002.

Saitou K, Washimi Y, Koike Y, Takahashi A, Kaneoke Y. Slow negative cortical potential preceding the onset of postural adjustment. Electroencephalogr Clin Neurophysiol 1996;98:449-455. [PubMed: 8763504]

Schepens B, Drew T. Independent and convergent signals from the pontomedullary reticular formation contribute to the control of posture and movement during reaching in the cat. J Neurophysiol 2004;92:2217-2238. [PubMed: 15175364]

Shibasaki H, Hallett M. What is the Bereitschaftspotential? Clin Neurophysiol 2006;117:2341-2356. [PubMed: 16876476]

Schneider C, Palomba D, Flor H. Pavlovian conditioning of muscular responses in chronic pain patients: central and peripheral correlates. Pain 2004;112:239-247. [PubMed: 15561378]

Shum GL, Crosbie J, Lee RY. Effect of low back pain on the kinematics and joint coordination of the lumbar spine and hip during sit-to-stand and stand-to-sit. Spine 2005;30:1998-2004. [PubMed: 16135992]

Shum GL, Crosbie J, Lee RY. Symptomatic and asymptomatic movement coordination of the lumbar spine and hip during an everyday activity. Spine 2005;30:E697-E702. [PubMed: 16319739] 
Siddall PJ, Stanwell P, Woodhouse A, Somorjai RL, Dolenko B, Nikulin A, Bourne R, Himmelreich U, Lean C, Cousins MJ, Mountford CE. Magnetic resonance spectroscopy detects biochemical changes in the brain associated with chronic low back pain: a preliminary report. Anesth Analg 2006;102:1164-1168. [PubMed: 16551917]

Tsao H, Galea MP, Hodges PW. Reorganization of the motor cortex is associated with postural control deficits in recurrent low back pain. Brain 2008;131:2161-2171. [PubMed: 18669505]

Tsao H, Hodges PW. Persistence of improvements in postural strategies following motor control training in people with recurrent low back pain. J Electromyogr Kinesiol 2008;18:559-567. [PubMed: 17336546]

Tsao H, Galea MP, Hodges PW. How fast are feedforward postural adjustments of the abdominal muscles? Behav Neurosci 2009;123:687-693. [PubMed: 19485575]

Van Dillen LR, Maluf KS, Sahrmann SA. Further examination of modifying patient preferred movement and alignment strategies in patients with low back pain during symptomatic tests. Man Ther. 2007 Electronic Publication, November 19.

Viallet F, Massion J, Massarino R, Khalil R. Coordination between posture and movement in a bimanual load lifting task: putative role of a medial frontal region including the supplementary motor area. Exp Brain Res 1992;88:674-684. [PubMed: 1587326]

Wiech K, Preissl H, Birbaumer N. Neuroimaging of chronic pain: phantom limb and musculoskeletal pain. Scand J Rheumatol Suppl 2000;113:13-18. [PubMed: 11028825]

Yoshida S, Nakazawa K, Shimizu E, Shimoyama I. Anticipatory postural adjustments modify the movement-related potentials of upper extremity voluntary movement. Gait Posture 2008;27:97-102. [PubMed: 17408954] 


\section{TIMING OF DEPENDENT MEASURES}
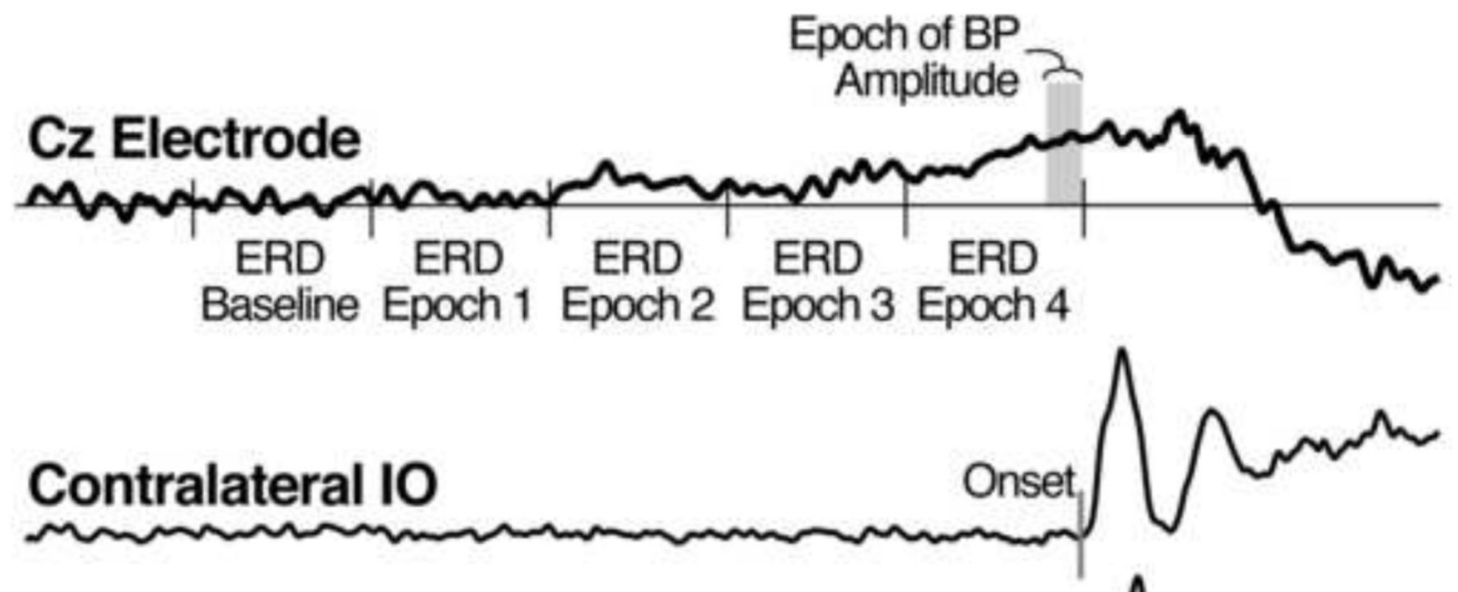

Contralateral ESP

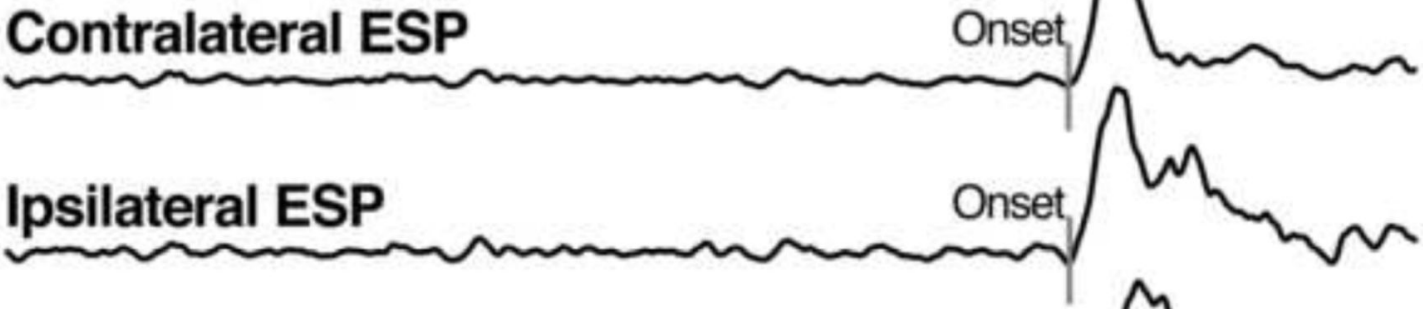

Ipsilateral IO

Deltoid

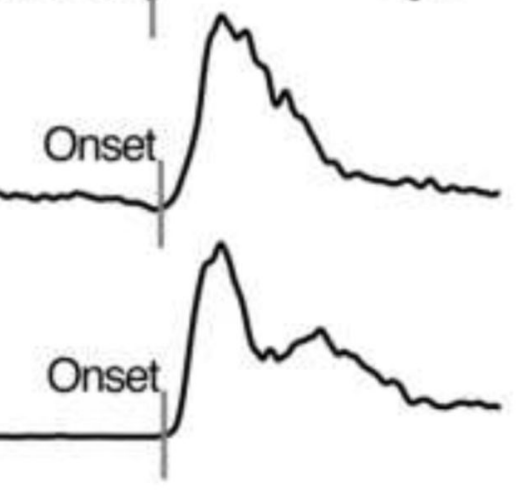

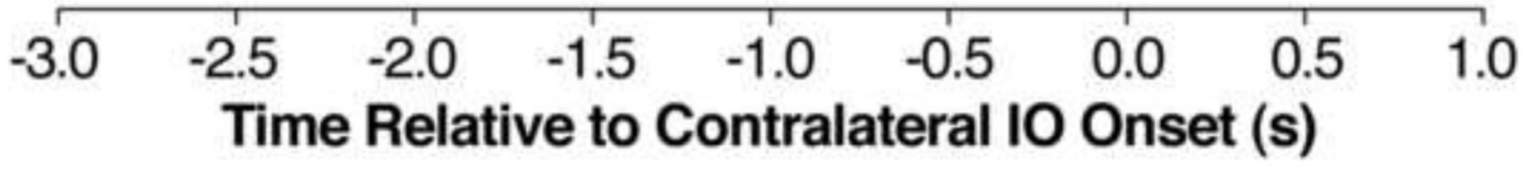

Fig. 1.

The relative timing of the selected dependent measures. Traces represent a representative participant's average traces, low-pass filtered to $15 \mathrm{~Hz}$ for illustration. The top trace represents an average EEG voltage signal from the $\mathrm{Cz}$ electrode, demonstrating the epochs in which mean alpha power and mean BP amplitudes were calculated. This trace is illustrated relative to the timing of activation onset for the deltoid (DELT), internal oblique (IO), and erector spinae (ESP) muscles. These muscle onset latencies appear similar only due to the lengthened time scale. 

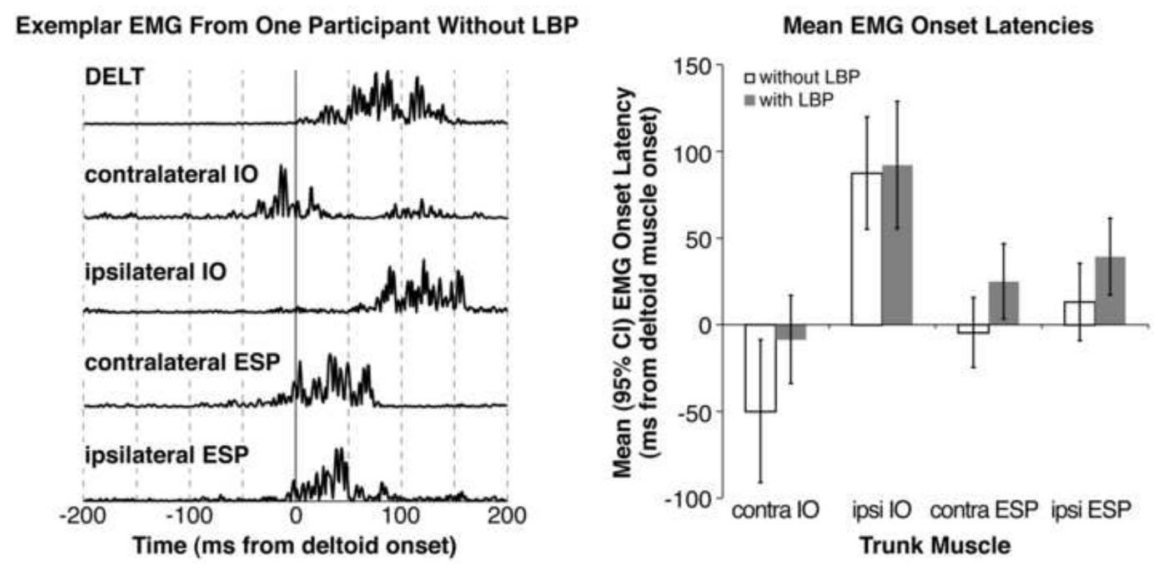

Fig. 2.

Mean EMG onset latencies for each group. The left chart illustrates the EMG activation of the DELT, IO, and ESP muscles from an example trial of a participant without LBP. The solid, black vertical line denotes the onset of the DELT muscle. The right chart illustrates the group mean $( \pm 95 \% \mathrm{CI})$ EMG onset latencies of the IO and ESP muscles contralateral (contra) and ipsilateral (ipsi) to the arm movement for the participants with LBP (gray bars) and those without LBP (open bars). 


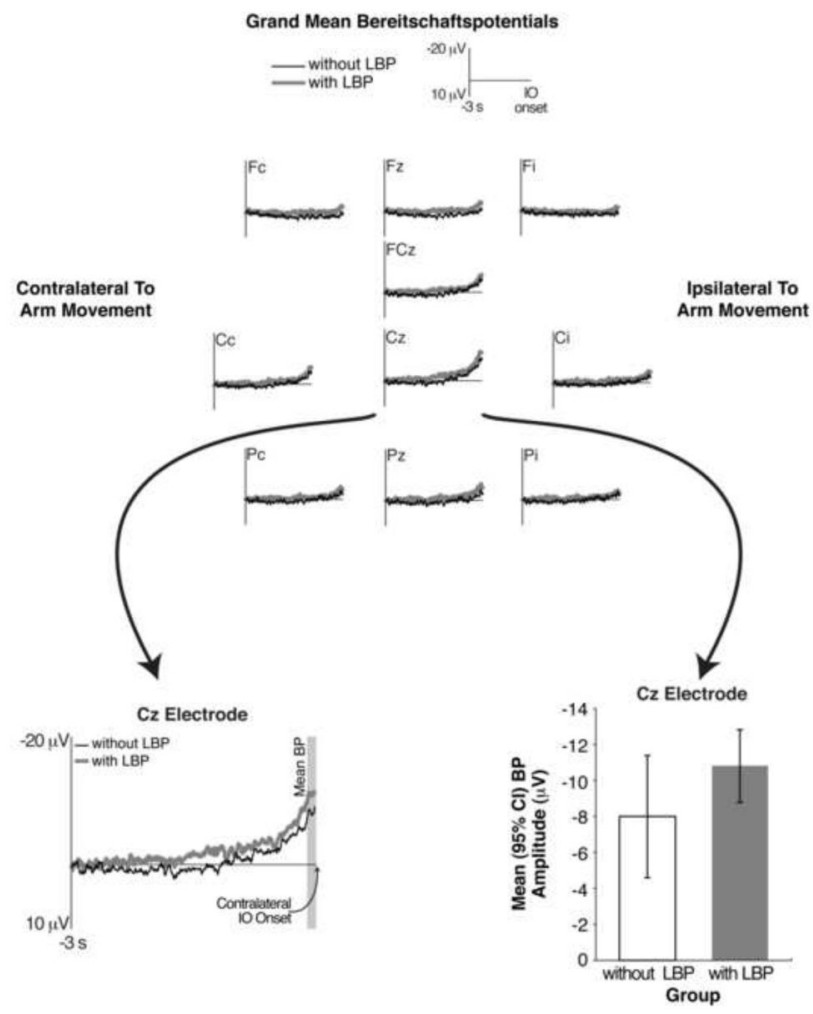

Fig. 3.

Grand mean Bereitschaftspotentials (BPs) for each group and electrode location. The top panel illustrates the grand mean EEG waveforms for participants with LBP (thick, gray traces) and those without LBP (thin, black traces) at each analyzed electrode location, with the Fc and Cc electrodes contralateral to the arm movement and the $\mathrm{Fi}$ and $\mathrm{Ci}$ electrodes ipsilateral to the arm movement. The bottom, left chart expands the traces from the $\mathrm{Cz}$ electrode to better illustrate the BP potential from its location of maximal amplitude as well as to illustrate the final 100 ms prior to the onset of the contralateral IO muscle (gray bar) from which mean BP amplitudes were derived for each participant. The bottom, right chart illustrates the grand mean $( \pm 95 \%$ CI) BP amplitudes from the $\mathrm{Cz}$ electrode for the participants with LBP (gray bar) and those without LBP (open bar). 

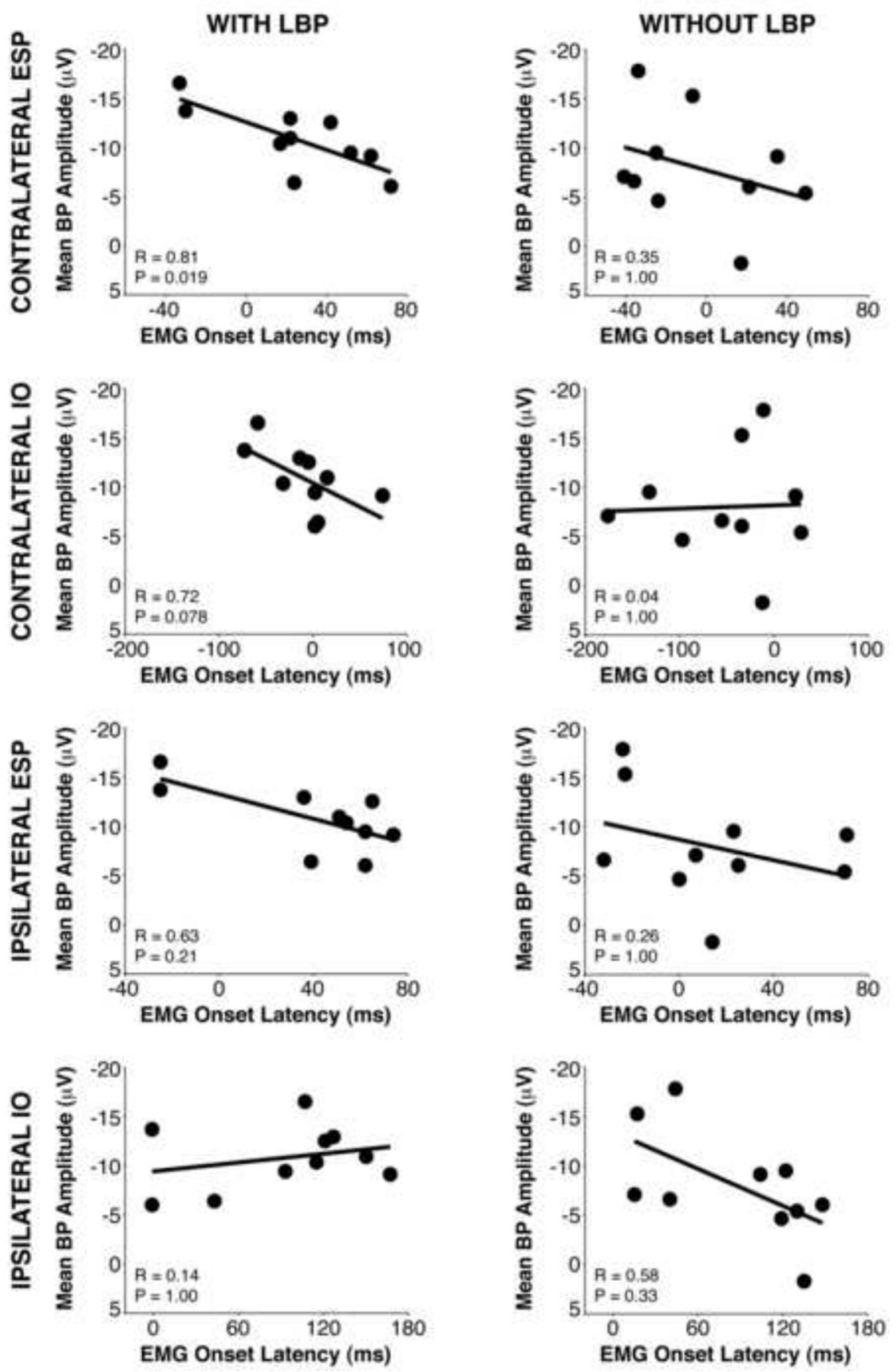

Fig. 4.

Associations among EMG onset latencies and BP amplitudes. The scatter plots illustrate the associations among the participants' IO and ESP muscle onset latencies with their mean BP amplitudes for those with LBP (left column) and those without LBP (right column). Circles denote values of individual participants and the solid lines denote the best-fit lines. The Rvalues represent the Spearman's correlation coefficients, and the P-values represent the Bonferroni-corrected levels of significance. Axes were kept constant to facilitate comparisons between the participant groups but differ for each muscle. 
GRAND MEAN ALPHA EVENT-RELATED DESYNCHRONIZATION
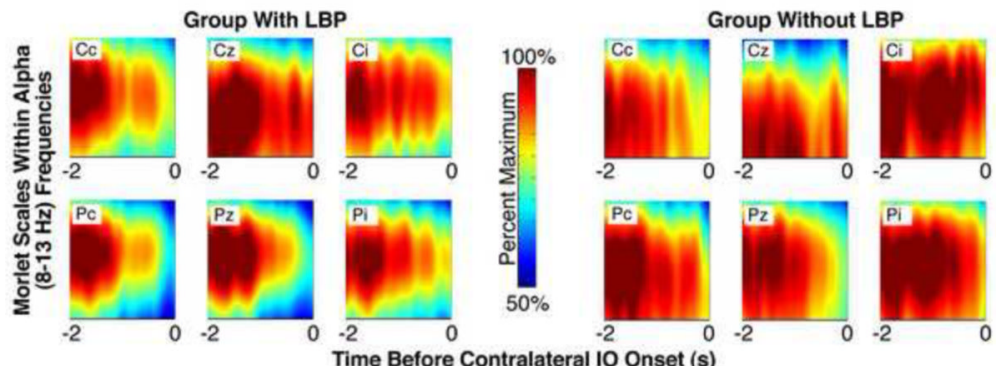

Group With LBP
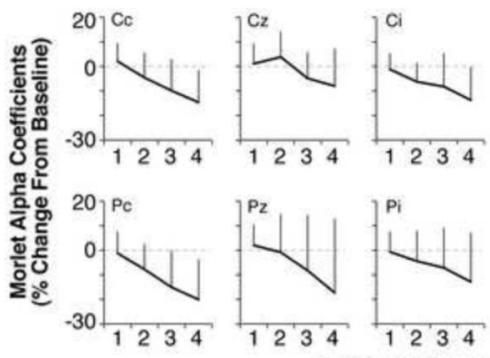

500-ms Interval Before Contralateral 10 Onset

Group Without LBP

Fig. 5.

Alpha event-related desynchronization (ERD) at central electrodes for each group. The 2dimensional shaded graphs demonstrate each group's grand mean of morlet coefficients (lighter shades illustrate higher coefficients; darker shades, lower coefficients) across the morlet scales within the alpha frequencies of $8-13 \mathrm{~Hz}$ (vertical axis; which has been reversed to reflect the pseudo-frequencies of the morlet output rather than the scales of the morlet output) over the $2000 \mathrm{~ms}$ that preceded activation of the contralateral IO muscle (horizontal axis). Color scales are equal for both groups. The Cc electrode represents the electrode contralateral to the arm movement; the $\mathrm{Ci}$ electrode, ipsilateral to the arm movement. The line charts illustrate each group's mean (standard deviation) binned and baseline-normalized alpha coefficients across the three central electrodes and the four successive 500-ms intervals that preceded activation of the contralateral IO muscle. The lines have been horizontally offset to facilitate visualization of each line. 
Table 1

Mean $( \pm 95 \%$ CI) Characteristics of Participants With and Without LBP

\begin{tabular}{|c|c|c|}
\hline \multirow{2}{*}{ Characteristic } & \multicolumn{2}{|c|}{ Group } \\
\hline & $\mathbf{L B P}(\mathbf{N}=\mathbf{1 0})$ & Control $(N=10)$ \\
\hline $\begin{array}{c}\text { Sex } \\
(\mathrm{N} \text { male, } \mathrm{N} \text { female })\end{array}$ & 5,5 & 5,5 \\
\hline $\begin{array}{l}\text { Age } \\
(\mathrm{yr})\end{array}$ & $39.20 \pm 6.33$ & $35.40 \pm 5.25$ \\
\hline $\begin{array}{l}\text { Height } \\
\text { (m) }\end{array}$ & $1.76 \pm 0.06$ & $1.81 \pm 0.20$ \\
\hline $\begin{array}{l}\text { Weight } \\
\quad(\mathrm{kg})\end{array}$ & $77.93 \pm 6.54$ & $70.92 \pm 6.55$ \\
\hline $\begin{array}{l}\text { Numeric Pain Rating Score } \\
\qquad(0-10 \text { scale })\end{array}$ & $1.78 \pm 0.92$ & $0.20 \pm 0.26$ \\
\hline $\begin{array}{c}\text { Oswestry Disability Score } \\
\text { (\% maximum) }\end{array}$ & $13 \pm 7$ & $0 \pm 0$ \\
\hline
\end{tabular}


Table 2

Mean $( \pm 95 \%$ CI $)$ of Each Group's Maximal Arm and Trunk Velocities

\begin{tabular}{|c|c|c|c|}
\hline \multirow{2}{*}{ Measure } & \multirow{2}{*}{ Trial Set } & \multicolumn{2}{|c|}{ Group } \\
\hline & & $\operatorname{LBP}(N=10)$ & Control $(\mathrm{N}=10)$ \\
\hline \multirow{2}{*}{$\begin{array}{l}\text { Sagittal Arm Velocity } \\
\qquad(\mathrm{m} / \mathrm{s})\end{array}$} & One & $2.51 \pm 0.39$ & $2.22 \pm 0.35$ \\
\hline & Three & $2.54 \pm 0.29$ & $2.72 \pm 0.37$ \\
\hline \multirow{2}{*}{$\begin{array}{l}\text { Frontal Trunk } \\
\text { Velocity }(\mathrm{m} / \mathrm{s})\end{array}$} & One & $0.074 \pm 0.030$ & $0.089 \pm 0.030$ \\
\hline & Three & $0.087 \pm 0.027$ & $0.112 \pm 0.025$ \\
\hline \multirow{2}{*}{$\begin{array}{l}\text { Sagittal Trunk } \\
\text { Velocity }(\mathrm{m} / \mathrm{s})\end{array}$} & One & $0.112 \pm 0.031$ & $0.118 \pm 0.030$ \\
\hline & Three & $0.131 \pm 0.027$ & $0.131 \pm 0.025$ \\
\hline
\end{tabular}

